\title{
Recurrent stroke in Neurosyphilis treated with intravenous fibrinolysis
}

\author{
Claudia Marando, Sara Mastrocola, David Giannandrea*, Silvia Cenciarelli and Stefano Ricci \\ Neurology Unit - Stroke Unit, Città di Castello Hospital, USL Umbria 1, Perugia, Italy
}

\section{Dear Editor}

We present the case of a 52-year-old caucasian man referred to our hospital with acute right-side paresis of arm and leg and ispilateral paresthesia, 25 minutes after symptoms onset. National Institute of Health Stroke Scale (NIHSS) score on admission was 4.

Patient's past medical history included nicotine consumption, diabetes mellitus, hypertension, hypercholesterolemia. Furthermore thirty days before presentation, he had experienced a left-sided hemispheric stroke, in the medial cerebral artery distribution, with three small fronto-parietal ischemic lesions detected by magnetic resonance imaging. This caused residual mild right lower limb weakness (modified Rankin Scale 1).

At the first hospitalization epiaortic Duplex Doppler ultrasound excluded hemodynamically significant stenosis. CT angiography revealed long soft atheroma plaque of carotid siphones, confirmed by brain angiography, causing $50 \%$ stenosis to the right one and $60 \%$ to the left one. Trans-esophageal echocardiogram and Holter electrocardiogram were normal. Routine laboratory tests including erythrocyte sedimentation rate, C-reactive protein, antinuclear and anti-DNA antibodies, antineutrophil cytoplasmic antibody, anticardiolipin antibodies, antinuclear antibody directed against extractable nuclear antigens and thrombophilic screening were normal. Infectious screening highlighted positive serum Venereal Disease Research Laboratory Results (VDRL, 1:128), T. pallidum haemagglutination (TPHA, 1:5120) and anti-treponema antibodies. The patient denied recent or remote history of any type of skin lesions, he reported unprotected sexual activity several years prior, but had been in a monogamous relationship with a female partner for six-years. Further serum testing provided a positive response to a rapid plasmin reagin test (RPR) at a 1:16 dilution, confirmed by a Treponema pallidum hemagglutination assay (TPPA) and fluorescent treponemal antibodyabsorption staining (FTA). Cerebrospinal fluid (CSF) analysis showed mild pleocytosis (30 cells, 50\% leukocytes), normal glucose $(93 \mathrm{mg} / \mathrm{dl}$ ) and protein levels $(35 \mathrm{mg} / \mathrm{dl})$. Results of a TPPA and FTA test of CSF were positive. Enzyme-linked immunoassay for HIV was negative. So we diagnosed vascular neurosyphilis and started antibiotic therapy with i.v. ceftriaxone 2 gr/day for two weeks.

At the second hospitalization he was regularly taking acetylsalicylic acid and statin. He also completed antibiotic therapy. A CT scan performed in emergency setting showed no signs of bleeding or hyperacute ischaemia. CT angiography confirmed stenosis of carotid siphones and highlighted high-grade narrowing of the left middle cerebral artery (section M3). Vital signs and blood tests on admission were within normal range. Despite the recent stroke, patient did not have any absolute exclusion criteria for the administration of rt-PA then, after obtaining written informed consent, it was given two hours after symptoms onset at the dose of $0.9 \mathrm{mg} / \mathrm{kg}$ (over 60 minutes as a $10 \%$ bolus and $90 \%$ infusion) with subsequent clinical improvement (NIHSS 1). The next day a magnetic resonance imaging of the brain showed a new small left hemispheric ischemic lesion without bleeding.

Neurosyphilis is defined as any involvement of the central nervous system (CNS) by syphilitic infection at any stage of infection and is classified into meningeal, vascular, and parenchymatous forms. The first and the second type often occur together, resulting in a "meningovascular neurosyphilis" [1]. Meningovascular syphilis occurs in $0,3-2,4 \%$ of all patients with syphilis [2] and usually occurs within 10 -years of infection $[1,3]$. The pathogenetic mechanism of vascular changes include diffuse thickening due to lymphocytic infiltration, fibroblastic and collegenous proliferation. In Heubner arteritis medium and large arteries are involved, while Nissl-Alzheimer arteritis affect small intracranial vessels [4]. The anterior circulation is more often involved, primarily in the territory of MCA; basilar artery with its branches are second most commonly involved $[4,5]$. Meningovascular syphilis is more likely to involve the supraclinoid portion of the internal carotid artery [1], as in our case.

Given the new increase in incidence of the disease, neurosyphilis should be considered in the differential diagnosis of ischaemic stroke, especially in young patients and in those without ischaemic risk factors. Due to the scarce data available, the optimal treatment of acute ischemic stroke in patients with neurosyphilis is uncertain. The use of i.v. thrombolysis in patients with vasculitis is controversial and should be regarded with caution because these patients might be at higher risk for secondary hemorrhagic conversion. CNS vasculitis can lead to intracerebral haemorrhage by weakening vessel walls and aneurysm formation. There is a lack of literature references for the use of i.v. rt-PA in patients presenting with acute ischemic stroke as the manifestation of meningovascular neurosyphilis. Also, there are no recommendations for the use of thrombolysis in such patients in international guidelines. However, several experts recommend to give thrombolysis if no absolute contraindications were present. Since the old lesions were small, there was no leucoaraiosis and no microbleeds on the first magnetic resonance, we decided to give thrombolysis with a very good clinical results.

${ }^{\star}$ Correspondence to: Giannandrea David, MD, U.O. Neurologia - Centro Ictus, Ospedale di Città di Castello, via L. Angelini n ${ }^{\circ} 10,06012$, Città di Castello (PG), Italy, E-mail: david.giannandrea@uslumbria1.it

Key words: stroke, neurosyphilis, intravenous thrombolysis

Received: March 09, 2018; Accepted: March 27, 2018; Published: March 29, 2018 
The present clinical case emphasizes the need to consider neurosyphilis in the differential diagnosis of ischaemic stroke in young adult patients, not always mentioned in national and international stroke guidelines.

\section{Declarations of interest}

\section{None}

\section{Source of funding}

This research received no specific grant from any funding agency in the public, commercial, or not-for-profit sectors.

\section{References}

1. Chahine LM, Khoriaty RN, Tomford WJ, Hussain MS (2011) The changing face of neurosyphilis. Int J Stroke 6: 136-143. [Crossref]

2. Gürses C, Bilgiç B, Topçular B, Tuncer OG, Akman-Demir G, et al. (2007) Clinica and magnetic resonance imaging findings of HIV-Negative patients with neurosyphilis. J Neurol 254: 368-374. [Crossref]

3. Simon RP (1985) Neurosyphilis. Arch Neurol 42: 606-613.

4. Bäuerle J, Zitzmann A, Egger K, Meckel S, Weiller C, et al. (2015) The great imitator - Still today! A case of meningovascular syphilis affecting the posterior circulation. $J$ Stroke Cerebrovasc Dis 24: e1-e3. [Crossref]

5. Han JH, Lee CC, Crupi RS (2004) Meningovascular syphilis and improvement with tissue-plasminogen activator (T-PA). Am J Emerg Med 22: 426-427. [Crossref]

Copyright: $(02018$ Marando C. This is an open-access article distributed under the terms of the Creative Commons Attribution License, which permits unrestricted use, distribution, and reproduction in any medium, provided the original author and source are credited. 